\title{
Atraso na busca por serviço de saúde para o diagnóstico da Tuberculose em Ribeirão Preto (SP)
}

\author{
Delay in the search for health services \\ for the diagnosis of tuberculosis in Ribeirao Preto, Sao Paulo
}

\author{
Aline Ale Beraldo ${ }^{1}$ \\ Tiemi Arakawa ${ }^{1}$ \\ Erika Simone Galvão Pinto ${ }^{2}$ \\ Rubia Laine de Paula Andrade ${ }^{1}$ \\ Anneliese Domingues Wysocki ${ }^{1}$ \\ Reinaldo Antonio da Silva Sobrinho ${ }^{3}$ \\ Beatriz Estuque Scatolin ${ }^{1}$ \\ Nathalia Hálax Órfão ${ }^{1}$ \\ Maria Amélia Zanon Ponce ${ }^{1}$ \\ Aline Aparecida Monroe ${ }^{1}$ \\ Lúcia Marina Scatena ${ }^{4}$ \\ Tereza Cristina Scatena Villa ${ }^{1}$
}

${ }^{1}$ Escola de Enfermagem de Ribeirão Preto,

Universidade de São Paulo. Av Bandeirantes 3900/ Campus USP, Monte

Alegre. 14040-902

Ribeirão Preto SP.

li_aab@yahoo.com.br

${ }^{2}$ Departamento de

Enfermagem, Universidade

Potiguar.

${ }^{3}$ Departamento de

Enfermagem, Universidade

Estadual do Oeste do

Paraná.

${ }^{4}$ Departamento de Medicina

Social, Universidade

Federal do Triângulo

Mineiro.

\begin{abstract}
The scope of this paper is to analyze delays in locating health services for the diagnosis of tuberculosis in Ribeirao Preto in 2009. An epidemiological and cross-sectional study was conducted with 94 TB patients undergoing treatment. A structured questionnaire, based on the Primary Care Assessment Tool adapted for TB care was used. A median (15 days or more) was established to characterize delay in health attendance. Using the Prevalence Ratio, the variables associated with longer delay were identified. The first healthcare services sought were the Emergency Services (ES) (57.5\%). The longest period between seeking assistance occurred among males, aged between 50 and 59, who earned less than five minimum wages, had pulmonary TB, were new cases, were not co-infected with TB/HIV, did not consume alcohol, had satisfactory knowledge about TB before diagnosis (with a statistically significant association with delay) and who did not seek healthcare close to home before developing TB. There is a perceived need for training healthcare professionals about the signs and symptoms of the disease, reducing barriers of access to timely diagnosis of $T B$ and widely disseminating it to the community in general.
\end{abstract}

Key words Tuberculosis, Late diagnosis, Access to health services
Resumo Foi analisado o atraso na busca por serviço de saúde para o diagnóstico da Tuberculose (TB) em Ribeirão Preto, 2009, em um estudo epidemiológico, transversal, realizado com 94 doentes. Utilizou-se questionário baseado no Primary Care Assessment Tool, adaptado para avaliar a atenção à TB. Estabeleceu-se a mediana (15 dias ou mais) para caracterizar atraso na busca pelo primeiro atendimento. Através da Razão de Prevalência, identificaram-se as variáveis relacionadas ao maior atraso. O primeiro serviço de saúde procurado foi o de pronto atendimento (57,4\%). Verificou-se um maior tempo na procura pelos serviços de saúde entre os doentes: do sexo masculino; com idade entre 50 a 59 anos; com renda familiar inferior a cinco salários mínimos; forma pulmonar; casos novos; não coinfecção TB/HIV; não consumiam bebida alcoólica, conhecimento satisfatório sobre TB (apresentou significância estatística na associação com o atraso) e que não procuravam o serviço de saúde próximo do domicílio antes de ter TB. Verificouse a necessidade de capacitação para os profissionais de saúde quanto aos sinais e sintomas da doença, reduzindo as barreiras de acesso ao diagnóstico oportuno da TB e divulgação ampla para a comunidade em geral.

Palavras-chave Tuberculose, Diagnóstico tardio, Acesso aos serviços de saúde 


\section{Introdução}

A tuberculose (TB) ainda aflige a humanidade, sendo considerada um problema de saúde pública não solucionado ${ }^{1}$. No que tange ao seu controle, o acesso aos serviços de saúde se configura como um desafio na realização do diagnóstico precoce, uma vez que, ao perceber-se doente, o indivíduo procede a busca e a utilização destes serviços, culminando no processo de oferta e recebimento de atenção à saúde. Este é resultante da interação do comportamento do indivíduo que procura cuidados e do profissional que o conduz dentro do sistema de saúde 2 .

No que se refere ao diagnóstico da TB, a conduta do doente em procurar serviços de saúde ainda é pouco conhecida ${ }^{3,4}$. No entanto, aspectos relativos aos serviços de saúde (como deficiência de recursos humanos, físicos e debilidades na oferta de atenção), bem como aqueles relacionados ao doente de TB (questões socioeconômicas, culturais) estão intimamente ligados e podem determinar o tempo decorrido no processo de diagnóstico da enfermidade em pauta.

Identificar o tempo de atraso do doente para o diagnóstico da TB (tempo que transcorre entre a percepção do indivíduo com relação aos sinais e sintomas, sentir-se doente e identificar a necessidade de buscar cuidados de saúde), transpondo obstáculos sociais, religiosos, pessoais e até mesmo físicos, certamente poderá auxiliar na elaboração de medidas para a localização oportuna das fontes de infecção ${ }^{5}$.

No Brasil, em estudo realizado na cidade de São Paulo o tempo de busca do doente pelo serviço de saúde foi maior que 30 dias $^{6}$. Em Gana, o referido atraso foi de até $30 \operatorname{dias}^{7}$ e na China, foi de 14 a 21 dias $^{8}$. Acredita-se que o tempo aceitável para um controle eficaz da TB não deve ser superior a 14 ou 21 dias $^{9}$, muito embora, na literatura, não haja concordância sobre o tempo aceitável para a busca pelo diagnóstico.

Neste contexto, estudar o tempo de atraso na busca por serviço de saúde para o diagnóstico da TB possibilitaria conhecer o comportamento dos doentes de TB nesta busca, contribuindo para a detecção precoce dos casos, e para o consequente rompimento da cadeia de transmissão da $\mathrm{TB}$, uma vez que cada doente com baciloscopia positiva sem tratamento infecta, em média, 8 a 15 contatos por ano ${ }^{10-11}$.

Diante do exposto, o presente estudo teve por objetivo analisar o atraso na busca por serviço de saúde para o diagnóstico da TB em Ribeirão Preto (SP), 2009.

\section{Material e métodos}

Estudo epidemiológico do tipo transversal, realizado em 2009, no município de Ribeirão Preto, cuja população era de 583.842 habitantes ${ }^{12}$. O sistema de saúde é constituído por 33 Unidades Básica de Saúde (UBS), 21 Unidades de Saúde da Família, 05 Unidades Básicas Distritais de Saúde (UBDS), com Serviços de Pronto-atendimento (SPA), que funcionam 24 horas, e 15 hospitais (totalizando 1942 leitos, sendo 1389 SUS e 553 conveniados) distribuídos nos cinco distritos sanitários do município ${ }^{13}$.

O manejo clínico dos doentes de TB é realizado em Ambulatórios de Referência para tratamento da doença. O município conta ainda com uma unidade especial de tratamento de moléstias infecciosas, alocada em um hospital estadual, que é responsável pelo manejo de casos mais complexos (coinfecção TB/HIV, multidrogarresistência e comorbidades). Em 2009, o município notificou 195 casos de TB e 19,6\% de coinfecção TB/HIV. Com $93,3 \%$ de cobertura de tratamento diretamente observado (TDO), o município atingiu $85,6 \%$ de taxa de cura, $8,7 \%$ de óbito, $3,6 \%$ abandono ${ }^{14}$.

A população de estudo foi constituída por 113 doentes de TB em tratamento no município no período de julho a novembro de 2009. Dentre estes doentes, dois não atendiam aos critérios de seleção (idade igual ou superior a dezoito anos, ser residente em Ribeirão Preto e estar fora do sistema prisional), quatro se recusaram a participar das entrevistas e seis não foram localizados mesmo com cinco tentativas de contato. Além disso, foram excluídos do estudo quatro doentes por não lembrar o tempo de busca do primeiro serviço de saúde quando da manifestação dos sintomas da TB e três por não saber qual o primeiro serviço de saúde procurado, o que resultou em uma população de 94 doentes.

Os dados foram coletados através de fonte primária (entrevista) e fontes secundárias (prontuários e sistemas de informação - TB-WEB), utilizando um questionário baseado no Primary Care Assessment Tool (PCAT) $)^{15}$, validado no Brasil $^{16}$ e adaptado para atenção à $\mathrm{TB}^{17}$, elencando para este estudo a dimensão porta de entrada $e$ acesso ao diagnóstico da Atenção Primária a Saú$\mathrm{de}^{15}$. Este instrumento elegeu questões que procuraram recuperar o trajeto do doente desde o momento em que apresentou sinais e sintomas da TB, sentiu-se doente e desejou obter cuidados específicos até a efetiva procura pelos serviços de saúde visando o diagnóstico. 
As variáveis dependentes utilizadas no estudo foram divididas em três seções: Informações sociodemográficas: sexo, idade, escolaridade, situação empregatícia e renda familiar; Informações Clínicas sobre o caso de TB (fonte secundária): forma clínica, coinfecção pelo HIV e tipo de caso; $D i$ mensão Porta de Entrada: realizava controle preventivo de saúde antes de adoecer de TB, procurava serviço de saúde mais próximo do domicílio, consumia bebida alcoólica, consumia tabaco, conhecimento sobre TB antes do diagnóstico, percepção quanto à gravidade dos sintomas, e primeiro serviço de saúde procurado quando se sentiu doente. Como variável independente, considerou-se o tempo (em dias) entre o início dos sinais e sintomas da TB e a primeira procura por atendimento, o qual determinou o atraso no diagnóstico da TB relacionado ao doente.

Para a análise dos dados, uma vez que a variável tempo entre o início dos sinais e sintomas da TB e a busca por atendimento possuía distribuição assimétrica, estabeleceu-se a mediana (15 dias) como um "ponto de corte" para a definição do que seria o atraso na busca por atendimento para o diagnóstico da TB. Ou seja, doentes que demoraram 15 dias ou mais para proceder à busca por um serviço de saúde após o início dos sinais e sintomas da doença foram considerados como atraso.

Posteriormente, calculou-se a Razão de Prevalência $(R P)^{18}$ para identificar as categorias das variáveis dependentes, que estiveram associadas ao atraso no diagnóstico da TB. Para a realização do cálculo da RP, os dados de cada variável utilizada foram alocados em tabelas $2 \mathrm{X} 2$ criando-se, assim, quatro grupos (Figura 1). Em se- guida, encontrou-se a RP por meio do cálculo da relação entre a proporção de doentes que tiveram atraso na categoria $1(\mathrm{a} / \mathrm{a}+\mathrm{b})$ e a proporção dos doentes que tiveram atraso na categoria 2 (c/ $\mathrm{c}+\mathrm{d}$ ). Padronizou-se como numerador a primeira categoria de cada variável. Foram construídos intervalos de confiança (IC95\%), adotando nível de significância de 5\% $(\alpha=0,05)$.

Quanto aos aspectos éticos, atendendo à resolução $196 / 96^{19}$, o estudo foi aprovado pelo Comitê de Ética da Escola de Enfermagem de Ribeirão Preto.

\section{Resultados}

A mediana do tempo entre o início dos sinais e sintomas da TB e a busca por um primeiro atendimento está apresentada na Figura 2. Em alguns casos foi observado que os doentes excederam 60 dias para proceder esta procura, tendo um doente relatado atraso de 1095 dias.

O primeiro serviço de saúde procurado foram os SPA $(57,4 \%)$, seguidos pelas Unidades de Atenção Básica (UAB) (24,5\%) e Serviços Especializados (SE) (18,1\%). O atraso na busca pelo primeiro atendimento ocorreu em $41(43,6 \%)$ dos 94 doentes entrevistados, sendo maior entre os doentes na faixa etária de 50 a 59 anos, que não consumiam bebidas alcoólicas, que não tinham o hábito de procurar o serviço mais próximo do domicílio antes de ficar doente e que tinham conhecimento satisfatório sobre a TB antes do diagnóstico, tendo este último resultado associação estatística significante com o atraso no diagnóstico (Tabela 1).

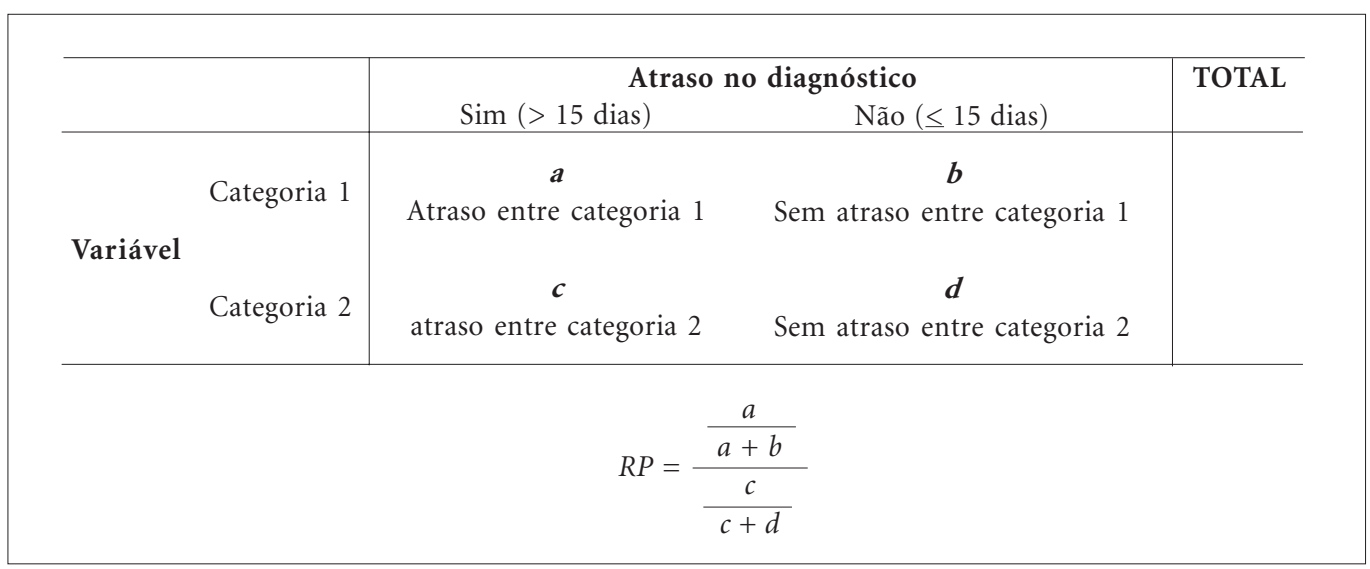

Figura 1. Esquema para o cálculo da razão de prevalência segundo o atraso no diagnóstico da tuberculose e variáveis selecionadas neste estudo. 
outros estudos realizados na China ${ }^{8,20}$ e na Tailândia ${ }^{21}$. Como justificativa para isto, os indivíduos alegaram falta de tempo ${ }^{8}$ por apoiarem economicamente a família e não estarem dispostos a negligenciar o referido papel assumido.

A busca por atendimento entre homens e mulheres tem sido explicada não só pelas diferen-

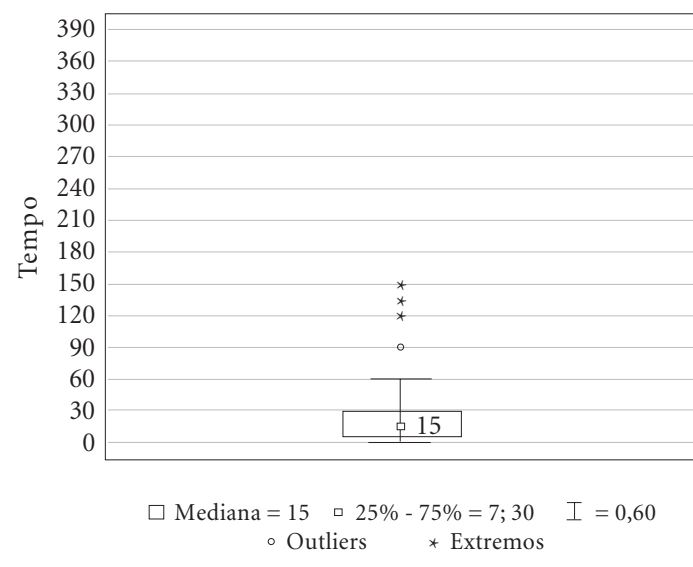

Figura 2. Tempo decorrido entre o início dos sintomas da Tuberculose e a procura pelo primeiro atendimento em um serviço de saúde no município de Ribeirão Preto, 2009.

\section{Discussão}

As escolhas individuais são cruciais na busca por cuidados de saúde e expressam construções subjetivas individuais (risco de adoecer e morrer, comportamento perante a doença) e também coletivas acerca do processo de adoecimento e da forma de buscar cuidados de saúde, forjadas sob as influências de diversos fatores e contextos. Neste sentido, nem todas as necessidades de saúde se convertem em demandas e nem todas estas são atendidas ${ }^{22}$, o que faz emergir a importância de um olhar para as necessidades dos doentes de TB no que tange ao acesso e à utilização dos serviços de saúde.

Em relação às informações sociodemográficas dos doentes de TB entrevistados, observou-se que nenhuma variável apresentou associação estatisticamente significante com o atraso na busca por serviço de saúde. No entanto, foi observado maior tempo (mediana) na faixa etária entre 50 a 59 anos, o que corrobora com resultados de ças do comportamento social, mas pelo modo como expressam a doença e pelos diferentes papéis que assumem em cada sociedade ${ }^{22}$. Esta diferença ainda se deve pelo perfil de necessidades de saúde, incluindo as demandas associadas à gravidez e ao parto $^{23}$ e também ao maior interesse das mulheres com relação à sua saúde, procurando serviços preventivos mais frequentemente ${ }^{24}$.

Estudos realizados no Brasil apontam que, de modo geral, a baixa adesão dos homens aos serviços de saúde está ligada a barreiras socioculturais, atreladas às posições assumidas pelo homem na sociedade, como o que é ser masculino ${ }^{25,26} \mathrm{e}$ como provedor de seu lar, também as barreiras institucionais, que restringem os horários de funcionamento dos serviços de saúde aos mesmos horários de trabalho destes indivíduos ${ }^{24}$ e nem sempre resolvem as demandas em uma única consulta $^{26-28}$. Verifica-se, no entanto, que atualmente, um grande número de mulheres se inseriu no mercado de trabalho e também são provedoras do lar, encontrando as mesmas barreiras para o acesso aos serviços de saúde e que, por este motivo, podem não estar se diferenciando dos homens na busca pelo diagnóstico da TB.

A baixa escolaridade, bem como a falta de provento e a baixa renda da maioria dos doentes de TB poderiam se configurar como uma dificuldade para a busca pelos serviços de saúde, no entanto, isto não foi verificado neste estudo, fato que poderia ser explicado pela maioria dos doentes entrevistados terem procurado serviços públicos e mais próximos de seu domicilio, não demandando, portanto, gastos com o atendimento e o transporte.

Em se tratando das informações clínicas dos doentes de TB, verificou-se um maior tempo para a busca por atendimento entre os doentes de TB pulmonar, no entanto esta diferença não foi estatisticamente significante. Estudo realizado mostra que doentes que apresentam tosse tem chance 11 vezes maior para o atraso na busca por serviço de saúde ${ }^{29}$, ou seja, estar com tosse, para alguns indivíduos, não significa estar doente ${ }^{30}$, adiando a busca pelo atendimento.

As experiências prévias com o serviço constituem da mesma forma uma influência sobre os comportamentos de busca por cuidado. Neste sentido, os casos novos de TB e os doentes que não apresentavam coinfecção $T B / H I V$ tiveram maior tempo na busca pelo primeiro serviço de saúde, assim como encontrado no estudo realizado por Díez et al. ${ }^{11}$, o que poderia estar relacionado ao fato dos casos em retratamento e de coinfecção com HIV já estarem atentos aos si- 
Tabela 1. Tempo decorrido entre o início dos sinais e sintomas da Tuberculose, distribuição de frequências e Razões de Prevalência do atraso do diagnóstico da doença, associados ao doente, segundo diferentes variáveis, Ribeirão Preto, 2009.

\begin{tabular}{|c|c|c|c|c|c|c|c|}
\hline & Variáveis & $\mathbf{N}(\%)$ & Mediana & Quartis & $\mathbf{N}$ & $\mathbf{R P}$ & IC \\
\hline \multirow{19}{*}{ 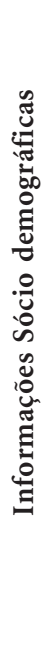 } & Sexo & & & & & & \\
\hline & Feminino & $29,0(30,9)$ & 14,0 & {$[7 ; 30]$} & 12 & 0,93 & {$[0,56-1,55]$} \\
\hline & Masculino & $65,0(69,1)$ & 15,0 & {$[7 ; 30]$} & 29 & & \\
\hline & Faixa Etária & & & & & & \\
\hline & 18 a 29 anos (1) & $24,0(25,5)$ & 18,0 & {$[7,45]$} & 12 & $\mathrm{RP} 1 / 2=1,11$ & {$[0,59-2,08]$} \\
\hline & 30 a $39 \operatorname{anos}(2)$ & $20,0(21,3)$ & 15,0 & {$[7 ; 30]$} & 09 & & \\
\hline & 40 a 49 anos (3) & $22,0(23,4)$ & 15,0 & {$[4 ; 30]$} & 07 & $\mathrm{RP} 1 / 3=1,57$ & {$[0,76-3,26]$} \\
\hline & 50 a 59 anos $(4)$ & $18,0(19,2)$ & 25,0 & {$[7 ; 30]$} & 10 & $\mathrm{RP} 1 / 4=0,90$ & {$[0,51-1,60]$} \\
\hline & Acima de 60 (5) & $10,0(10,6)$ & 3,0 & {$[1 ; 20]$} & 03 & $\mathrm{RP} 1 / 5=1,67$ & {$[0,60-4,66]$} \\
\hline & Escolaridade & & & & & & \\
\hline & Sem Escolaridade ou EF incompleto & $61,0(64,9)$ & 15,0 & {$[7 ; 30]$} & 14 & 0,96 & {$[0,59-1,56]$} \\
\hline & EF completo ou mais & $33,0(35,1)$ & 15,0 & {$[3 ; 30]$} & 27 & & \\
\hline & Situação Empregatícia & & & & & & \\
\hline & Com remuneração & $60,0(63,8)$ & 15,0 & {$[5 ; 30]$} & 26 & 0,98 & {$[0,61-1,58]$} \\
\hline & Sem remuneração & $34,0(36,2)$ & 15,0 & {$[7 ; 30]$} & 15 & & \\
\hline & Renda Familiar & & & & & & \\
\hline & Até 2 SM (1) & $57,0(60,6)$ & 15,0 & {$[6 ; 30]$} & 27 & $\mathrm{RP} 1 / 2=1,22$ & {$[0,73-2,06]$} \\
\hline & De $2 \mathrm{SM}$ a $5 \mathrm{SM}(2)$ & $31,0(33,0)$ & 15,0 & {$[7 ; 30]$} & 12 & & \\
\hline & Acima de 5 SM (3) & $6,0(6,4)$ & 9,0 & {$[3 ; 60]$} & 02 & $\mathrm{RP} 1 / 3=1,42$ & {$[0,44-4,55]$} \\
\hline \multirow{11}{*}{ 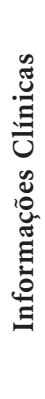 } & Forma Clínica da Doença & & & & & & \\
\hline & Pulmonar (1) & $75,0(79,8)$ & 15,0 & {$[7 ; 30]$} & 36 & $\mathrm{RP} 1 / 2=1,92$ & {$[0,80-4,63]$} \\
\hline & Extrapulmonar (2) & $16,0(17,0)$ & 14,0 & {$[3 ; 23]$} & 04 & & \\
\hline & Pulmonar + extrapulmonar (3) & $3,0(3,2)$ & 7,0 & {$[0 ; 30]$} & 01 & $\mathrm{RP} 1 / 3=1,44$ & {$[0,29-7,26]$} \\
\hline & Tipo de caso & & & & & & \\
\hline & Casos novos (1) & $87,0(92,6)$ & 15,0 & {$[7 ; 30]$} & 39 & $\mathrm{RP} 1 / 2=1,12$ & {$[0,37-3,36]$} \\
\hline & Recidiva/retratamento (2) & $5,0(5,3)$ & 3,0 & {$[2 ; 20]$} & 02 & & \\
\hline & Tratamento após Abandono (3) & $2,0(2,1)$ & 0,0 & {$[0 ; 0]$} & 00 & $\mathrm{RP} 1 / 3=0,00$ & -- \\
\hline & Co-infecção TB/HIV & & & & & & \\
\hline & Sim & $15,0(16,0)$ & 7,0 & {$[2 ; 15]$} & 03 & 0,42 & {$[0,14-1,17]$} \\
\hline & Não & $79,0(84,0)$ & 15,0 & {$[7 ; 30]$} & 38 & & \\
\hline \multirow{19}{*}{ 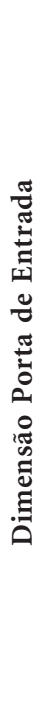 } & Sintomas da doença & & & & & & \\
\hline & Forte (1) & $67,0(71,3)$ & 15,0 & {$[7 ; 30]$} & 29 & $\mathrm{RP} 1 / 2=0,99$ & {$[0,53-1,84]$} \\
\hline & Moderado (2) & $16,0(17,0)$ & 15,0 & {$[2,5 ; 30]$} & 07 & & \\
\hline & Fraco $(3)$ & $11,0(11,7)$ & 15,0 & {$[2 ; 30]$} & 05 & $\mathrm{RP} 1 / 3=0,95$ & {$[0,47-1,92]$} \\
\hline & Realização de Controle Preventivo de S & & & & & & \\
\hline & Sim & $57,0(60,6)$ & 15,0 & {$[7 ; 30]$} & 21 & 0,68 & {$[0,43-1,07]$} \\
\hline & Não & $37,0(39,4)$ & 15,0 & {$[4 ; 30]$} & & & \\
\hline & Consumo de bebidas alcoólicas & & & & & & \\
\hline & Sim & $74,0(78,7)$ & 15,0 & {$[5 ; 30]$} & 29 & 0,65 & {$[0,41-1,03]$} \\
\hline & Não & $20,0(21,3)$ & 20,0 & {$[7 ; 38]$} & 12 & & \\
\hline & Consumo de Tabaco & & & & & & \\
\hline & Sim & $61,0(64,9)$ & 15,0 & {$[7 ; 30]$} & 27 & 1,04 & {$[0,64-1,70]$} \\
\hline & Não & $33,0(35,1)$ & 15,0 & {$[3 ; 30]$} & 14 & & \\
\hline & Conhecimento sobre a TB antes do dia & & & & & & \\
\hline & Precário & $62,0(66,0)$ & 15,0 & {$[7 ; 30]$} & 22 & 0,60 & {$[0,38-0,93]^{*}$} \\
\hline & Satisfatório & $32,0(34,0)$ & 21,0 & {$[5 ; 30]$} & 19 & & \\
\hline & $\begin{array}{l}\text { Procura por SS mais próximo do domic } \\
\text { de ficar doente TB }\end{array}$ & & & & & & \\
\hline & Sim & $66,0(70,2)$ & 15,0 & {$[7 ; 30]$} & 27 & 0,82 & {$[0,51-1,31]$} \\
\hline & Não & $28,0(28,8)$ & 18,0 & {$[3 ; 30]$} & 14 & & \\
\hline
\end{tabular}

Legenda: N (número total de doentes); n (número de doentes com atraso); UAB (Unidades de Atenção Básica - compreende as Unidades básicas de saúde e unidades de saúde da família; SPA (Serviços de Pronto Atendimento); SE (Serviços Especializados - compreende os hospitais, consultórios particulares e ambulatórios de referência); EF (Ensino Fundamental); SM (Salário Mínimo); TB (Tuberculose) e HIV (Human Immunodeficiency Virus); RP1/n razão de prevalência entre a categoria 1 e a categoria n, n variando de 1 a 5 ; *apresentou significância estatisticamente significativa. 
nais e sintomas da doença e/ou estarem em acompanhamento sistemático, demandando cuidado em tempo oportuno.

Considerando a dimensão Porta de Entrada, nenhuma variável apresentou associação estatisticamente significante com o atraso na busca por atendimento, exceto o "conhecimento sobre a TB antes do diagnóstico". Neste caso, os indivíduos que tinham conhecimento satisfatório sobre a doença foram os que mais atrasaram na busca por serviços de saúde, podendo evidenciar que conhecimento cognitivo nem sempre leva o indivíduo a tomar uma atitude para buscar um serviço de saúde. Diferentemente do que ocorreu em outro estudo ${ }^{31}$, uma questão que poderia explicar este achado se fundamenta na relação entre o conhecimento e os aspectos culturais, como o estigma, de modo que pessoas com maior conhecimento poderiam identificar em si os sinais e os sintomas da TB e sentir medo de buscar um serviço de saúde, por receio de ter uma doença infecciosa, transmissível e com tratamento longo, como também das pessoas descobrirem que está doente ${ }^{32}$.

Apesar do estudo identificar que o maior atraso ocorreu entre aqueles com conhecimento satisfatório sobre a doença, este era precário na maior parte dos entrevistados, condizente com outros estudos realizados em São Paulo ${ }^{6}$ e na Tanzânia ${ }^{33}$. Neste sentido, destaca-se a importância do conhecimento sobre a TB e seu tratamento, o acesso às informações através dos meios de comunicação, das escolas e de intervenções educativas para reduzir o estigma e o impacto das consequências sociais da TB, assim como programas de educação continuada de saúde projetados para aumentar o nível de conhecimento em toda a população como forma de melhorar o acesso dos doentes aos serviços de saúde para o diagnóstico oportuno da doença.

Quanto à gravidade dos sintomas da doença e a realização de controle preventivo de saúde, não foram verificadas diferenças no tempo de busca por serviços de saúde entre os grupos estudados. Em função destes resultados, cogita-se a possibilidade destes indivíduos não estarem aptos a distinguir e a caracterizar a gravidade de seus sintomas, bem como a possibilidade dos serviços de saúde não estarem capacitando seus usuários para tal. Destaca-se ainda a importância da integração entre as áreas de prevenção e controle para a identificação dos doentes de TB, culminando em um diagnóstico precoce, o que minimizaria a cadeia de transmissão da doença ${ }^{34}$.

Hábitos de vida como o consumo de bebida alcoólica e de tabaco podem estar associados à
TB pulmonar ${ }^{35,36}$, bem como ao maior atraso na busca pelos serviços ${ }^{37-39}$. No entanto, neste estudo, não foram observadas diferenças entre os grupos, evidenciando um possível viés de informação, visto que questionou-se apenas o uso de bebidas alcoólicas e de tabaco, não investigando frequência e consumo diário.

Verificou-se ainda um menor tempo de busca por atendimento entre doentes que tinham o hábito de procurar pelo serviço de saúde mais próximo do domicílio antes da TB, corroborando com estudo realizado no Vietnã ${ }^{40}$. Aspectos que podem estar relacionado à preferência do usuário por determinados serviços referem-se à satisfação com o atendimento anteriormente prestado $^{41}$, bem como ao vínculo estabelecido entre o usuário e o serviço de saúde ${ }^{15}$.

Neste sentido, a escolha do primeiro serviço de saúde para atendimento, que neste estudo foi em sua maioria os SPA, poderia estar pautada nas referidas experiências prévias, bem como em aspectos organizacionais (disponibilidade imediata de consultas médicas/exames diagnósticos e horário de funcionamento) e culturais ${ }^{32}$ (crença em uma maior resolutividade daqueles que dispõe de maior densidade tecnológica).

Frente ao exposto, acredita-se que o acesso aos serviços de saúde perpassa por barreiras que avançam os aspectos abordados neste estudo, e, desta forma, questões de ordem cultural e organizacional podem se constituir em variáveis de confundimento para a determinação da busca por atendimento. Diante disto, identificou-se a importância da realização de novos estudos com delineamento capaz de controlar estas variáveis, além de minimizar um possível viés de memória decorrido do fato de o doente não precisar a data do início dos sintomas e da busca pelo serviço de saúde.

\section{Considerações finais}

Verificou-se a necessidade de capacitar os profissionais de saúde para os sinais e os sintomas da TB e reforçar atividades de busca ativa na comunidade. Além disso, faz-se necessário a desmistificação da doença, através de informações sobre a TB nos distintos espaços sociais, posto que aspectos culturais podem estar interferindo na busca por atendimento, bem como na identificação dos casos suspeitos da doença, reduzindo assim as barreiras de acesso aos serviços de saúde e por consequência as barreiras ao diagnóstico oportuno da TB. 


\section{Colaboradores}

AA Beraldo, participou da concepção, delineamento, análise e interpretação dos dados, redação do artigo, revisão crítica e da aprovação da versão a ser publicada; T Arakawa, participou da revisão crítica; ESG Pinto, participou da revisão crítica; RLP Andrade, participou da revisão crítica e da aprovação da versão a ser publicada; $A D$ Wysocki, participou da revisão crítica; RA Silva Sobrinho, participou da revisão crítica; BE Scatolin, participou da revisão crítica; NH Órfão, participou da revisão crítica; MAZ Ponce, participou da revisão crítica; AA Monroe, participou da revisão crítica; LM Scatena, participou da análise e interpretação dos dados; TCS Villa, participou da concepção, delineamento, revisão crítica e da aprovação da versão a ser publicada .

\section{Agradecimentos}

A pesquisa realizada foi financiada pela Fundação de Amparo à Pesquisa de São Paulo (FAPESP) e pelo Conselho Nacional de Desenvolvimento Científico e Tecnológico (CNPq). Tratase de uma pesquisa, que faz parte de um projeto multicêntrico intitulado "Retardo no diagnóstico da tuberculose: análise das causas em diferentes regiões do Brasil".

\section{Referências}

1. Cavalcanti ZR, Albuquerque MFPM, Campello ARL, Ximenes R, Montarroyos U, Verçosa MKA. Características da tuberculose em idosos no Recife (PE): contribuição para o programa de controle. $J$ Bras Pneumol 2006; 32(6):535-543.

2. Travassos C, Martins M. Uma revisão sobre os conceitos de acesso e utilização de serviços de saúde. Cad Saude Publica 2004; 20(Supl. 2):S190-S198.

3. Goldbaum M, Gianini RJ, Novaes HMD, Galvão César CL. Utilização de serviços de saúde em áreas cobertas pelo programa saúde da família (Qualis) no Município de São Paulo. Rev Saude Publica 2005; 39(1):90-99.

4. Mendoza-Sassi R, Béria JU. Utilización de los serviços de salud: una revisión sistemática sobre los factores relacionados. Cad Saude Publica 2001; 17(4):819-832.

5. Sherman LF, Fujiwara PI, Cook SV, Bazerman LB, Frieden TR. Patient and health care system delays in the diagnosis and treatment of tuberculosis. Int $J$ Tuberc Lung Dis 1999; 3(12):1088-1095.

6. Job JRPP, Gozzano JOA, Bernardes Júnior OR, Garcia RH, Miralhes OJC, Miranda MAP. Informações que antecederam o diagnóstico de tuberculose pulmonar e tempo decorrido até o início do tratamento em pacientes matriculados em Centro de Saúde, São Paulo (Brasil). Rev Saude Publica 1986; 20(1):21-25.

7. Lawn SD, Afful B, Acheampong JW. Pulmonary tuberculosis: diagnostic delay in Ghanaian adults. Int J Tuberc Lung Dis 1998; 8(2):635-640.

8. Cheng G, Tolhurst RZL, Meng QY, Tang S. Factors affecting delays in tuberculosis diagnosis in rural China: A case study in four counties in Shandong Province. Trans R Soc Trop Med Hyg 2005; 99(5):355362.

9. Lamber TML, Stuyft P. Delays to tuberculosis treatment: shall we continue to blame the victim? Trop Med Int Health 2005; 10(10):945-946.

10. Cáceres-Manrique FM, Orozco-Vargas LC. Demora en el diagnóstico de tuberculosis pulmonar en una región de Colombia. Rev Salud Publica (Bogota) 2008; 10(1):94-104.

11. Díez M, Bleda MJ, Alcaide J, Castells C, Cardenal JI, Domínguez A, Gayoso P, Guitiérrez G, Huerta C, López MJ, Moreno T, Muñoz F, García-Fulgueiras A, Picó M, Pozo F, Quirós JR, Robles F, Sánchez JM, Vanaclocha H, Vega T. Multicentre Project for Tuberculosis Research (MPTR) Study Group. Determinants of health system delay among confirmed tuberculosis cases in Spain. Eur J Public Health 2005; 15(4):343-349.

12. Instituto Brasileiro de Geografia e Estatística (IBGE). Estimativas das populações residentes, segundo município. [acessado 2010 nov 30]. Disponível em: http:/ /www.ibge.gov.br/home/estatistica/populacao/ estimativa2009/POP2009_DOU.pdf

13. Ribeirão Preto. Secretaria Municipal de Saúde. Relatório de Gestão 2009. 2009. [acessado 2011 jan 05]. Disponível em: http://www.ribeiraopreto.sp. gov.br/ssaude/conselho/i16relatorio-gestao-09.pdf 
14. TB-WEB. Sistema de Notificação e Acompanhamento dos Casos de Tuberculose. [acessado 2010 mar 17]. Disponível em: http://www.cvetb.saude.sp.gov.br/ tbweb/index.jsp. (acesso restrito).

15. Starfield B. Atenção primária: equilíbrio entre necessidades de saúde, serviços e tecnologia. Brasília: Unesco; 2002.

16. Macinko J, Almeida C. Validação de uma metodologia de avaliação rápida das características organizacionais e do desempenho dos serviços de atenção básica do Sistema Único de Saúde (SUS) em nível local. Brasília: Organização Pan-Americana da Saúde; 2006.

17. Villa TCS, Ruffino-Netto A. Questionário para avaliação de desempenho de serviços de atenção básica no controle da TB no Brasil. J. Bras. Pneumol 2009; 35(6):610-612.

18. Passos ADC, Ruffino-Netto A. Estudos Transversais. In: Franco LJ, Passos ADC, organizadores. Fundamentos da Epidemiologia. Barueri: Manole; 2011.

19. Brasil. Ministério da Saúde (MS). Conselho Nacional de Saúde. Resolução no. 196/1996. Diretrizes e Normas Regulamentadoras de Pesquisas Envolvendo Seres Humanos. Diário Oficial da União 1996; out 10 .

20. Xu B, Jiang Q-W, Diwan VK. Diagnostic delays in access to tuberculosis care in counties with or without the National Tuberculosis Control Programme in rural China. Int J Tuberc Lung Dis 2005; 9(7):784790 .

21. Rojpibulstit M, Kanjanakiritamrong J, Chongsuvivatwong V. Patient and health system delays in the diagnosis of tuberculosis in Southern Thailand after health care reform. Int J Tuberc Lung Dis 2006; 10(4):422-428.

22. Travassos C, Viacava F, Fernandes C, Almeida M. Desigualdades geográficas e sociais na utilização de serviços de saúde no Brasil. Cien Saude Colet 2000; 5(1):133-149.

23. Pinheiro RS, Viacava F, Travassos C, Brito AS. Gênero, morbidade, acesso e utilização de serviços de saúde no Brasil. Cien Saude Colet 2002; 7(4):687-707.

24. Verbrugge LM. The twain meet: empirical explanations of sex differences in health and mortality. $J$ Health Soc Behav 1989; 30(3):282-304.

25. Gomes R. Sexualidade masculina e saúde do homem: proposta para uma discussão. Cien Saude Colet 2003; 8(3):825-829.

26. Schraiber LB, Gomes R, Couto MT. Homens e saúde na pauta da saúde coletiva. Cien Saude Colet 2005; 10(1):7-17.

27. Brasil. Ministério da Saúde (MS). Secretaria de Atenção à saúde. Departamento de ações Programáticas Estratégicas Política nacional de atenção integral à saúde dos homens: princípios e diretrizes. Brasília: MS; 2008.

28. Gomes R, Nascimento EF, Araújo FC. Por que os homens buscam menos os serviços de saúde do que as mulheres? As explicações de homens com baixa escolaridade e homens com ensino superior. Cad Saude Publica 2007; 23(3):565-574.

29. Sreeramareddy CT, Panduru KV, Menten J, Ende JVD. Time delays in diagnosis of pulmonary tuberculosis: a systematic review of literature. BMC Infect Dis 2009; 9:91-101.
30. Nogueira JA, Ruffino Netto A, Monroe AA, Gonzales RIC, Villa TCS. Busca ativa de sintomáticos respiratórios no controle da tuberculose na percepção do agente comunitário de saúde. Revista Eletrônica de Enfermagem 2007; 9(1):106-118.

31. Ayuo PO, Diero WD, Owino-Ong'OR, Mwangi AW. Causes of delay in diagnosis of pulmonary tuberculosis in patients attending a referral hospital in western Kenya. East Afr Med J 2008; 85(6):263-268.

32. Oliveira MF, Arcêncio RA, Ruffino Netto A, Scatena LM, Palha PF, Villa TCS. A porta de entrada para o diagnóstico da tuberculose no sistema de saúde de Ribeirão Preto/SP. Rev Esc Enferm USP 2001; 45(4):898-904.

33. Wandwalo ER, Morkve O. Knowledge of disease and treatment among tuberculosis patients in Mwanza, Tanzania. Int J Tuberc Lung Dis 2000; 4(11):10411046.

34. Silva TPT, Ferreira ILM. Doenças infecciosas e parasitárias: guia de bolso. Cad Saude Publica 2006; 22(11):2498.

35. Caron Ruffino M, Ruffino Netto A. Associação entre alcoolismo e Tuberculose Pulmonar. Rev Saude Publica 1979; 13(3):183-194.

36. Altet-Gómez MN, Alcaide J, Godoy P, Romero MA, Hernández DRI. Clinical and epidemiological aspects of smoking and tuberculosis: a study of 13038 cases. Int J Tuberc Lung Dis 2004; 9(4):430-436.

37. Saqib AM, Awan IN, Rizvi SK, Shahzad MI, Mirza ZS, Tahseen S, Khan IH, Khanum A. Delay in diagnosis of tuberculosis in Rawalpindi, Pakistan. BMC Res Notes 2011; 4(165):01-05.

38. Basnet R, Hinderaker SG, Enarson D, Malla P, Morkve O. Delay in the diagnosis of tuberculosis in Nepal. BMC Public Health 2009; 9:236-245.

39. Díez M, Bleda MJ, Alcaide J, Caloto T, Castells C, Cardenal JI, Domínguez A, Gayoso P, Gutiérrez G, Huerta C, López MJ, Moreno T, Muñoz F, Navarro C, Picó M, Pozo F, Quirós JR, Robles F, Sánchez JM, Vanaclocha H, Vega T, and Multicentre project for tuberculosis research (MPTR) study group. Determinants of patient delay among tuberculosis cases in Spain. Eur J Public Health 2004; 14(2):151-155.

40. Huong NT, Vree M, Duong BD, Khanh VT, Loan VT, Co NV, Borgdorff MW, Cobelens FG. Delays in the diagnosis and treatment of tuberculosis patients in Vietnam: a cross-sectional study. BMC Public Health 2007; 7(110):01-08.

41. Lafaiete RS, Motta MCS, Villa TCS. Satisfação no programa de controle da tuberculose em um município do Rio de Janeiro, Brasil. Rev Lat Am Enfermagem 2011; 19(3):[07 telas].

Artigo apresentado em 30/04/2012

Aprovado em 17/07/2012

Versão final apresentada em 30/08/2012 